\title{
The chemical composition towards the Galactic anti-centre. I. Observations and model atmosphere analyses
}

\author{
S.J. Smartt, P.L. Dufton and W.R.J. Rolleston \\ The Department of Pure and Applied Physics, The Queen's University of Belfast, Belfast BT7 1NN, N. Ireland
}

Received July 24; accepted October 14, 1995

\begin{abstract}
High resolution spectra of six early B-type main-sequence stars having galactocentric distances of between 10 and $18 \mathrm{kpc}$ are presented. We list the equivalent widths for the metal lines and illustrate their hydrogen and helium line profiles. The stars are analysed using LTE line-blanketed model atmosphere techniques to derive atmospheric parameters and surface chemical compositions. All six stars have similar effective temperatures and surface gravities, allowing a reliable comparison of their metal abundances and distances. Significant variations in the photospheric abundances are evident and we discuss the need for a more detailed line-by-line differential analysis to exactly quantify the differences. This will be presented in a companion paper (Smartt et al. 1996).
\end{abstract}

Key words: stars: abundances — stars: early-type — Galaxy: abundances

\section{Introduction}

Previous studies of the chemical composition of the Galaxy using different methods have led to conflicting results. We are engaged in a project to search for galactic abundance variations in both the solar neighbourhood and beyond. These studies are based on model atmosphere analyses of early B-type stars, in the field and young open clusters, which exist at different spatial positions and galactocentric distances. The derived photospheric abundances should reflect those of the present day interstellar medium, since our targets are normally on the main-sequence and hence should not be affected by any nuclear processed material from their cores. Also, being young (with typical ages of 10 million years) they should not have moved far from their birthplace.

This paper is the first of two in which we present high quality CCD spectra of a set of six stars in the Galactic anti-centre direction. All six targets have similar atmospheric parameters and they constitute a self-consistent data set allowing accurate and meaningful comparisons of their photospheric abundances. The spectra are analysed, using similar spectral features, by line-blanketed LTE model atmosphere techniques to derive the atmospheric parameters and absolute chemical compositions. Systematic errors existing in the methods of analysis should therefore be minimised when comparing the results. We also include the analysis of a suitable comparison star S285-6 (Rolleston et al. 1994), which has an effective tem-

Send offprint requests to: S.J. Smartt perature and logarithmic surface gravity similar to our programme stars. The discrepancies in the results for stars both studied here and by other authors (Kaufer et al. 1994 and Kilian-Montenbruck et al. 1996) are discussed.

In the second paper (Smartt et al. 1996, hereafter Paper II), we present a differential line-by-line abundance analysis of the target stars with respect to S285-6, which should more reliably identify variations in metal abundances. We also calculate reliable galactic positions while discussing the sources of error in the distance determinations. These results are discussed in the context of previous work in this area, including $\mathrm{H}$ II regions, planetary nebulae and other B-star studies in an attempt to draw a clearer picture of the variations in the chemical composition of the Galaxy beyond the solar circle.

\section{Observational data}

Our individual targets associated with the $\mathrm{H}$ II regions S208, S289 and S283 (as catalogued by Sharpless 1959) were selected on the basis of their photo-electric $U B V$ colours discussed by Moffat et al. (1979). The photometry indicated that these are OB main-sequence stars with similar atmospheric parameters and spectral types between 09.5 and B1. The two RLWT stars were selected from a list of 14 targets studied photometrically by Philip et al. (1990), who confirmed their classification as early-type main-sequence B-stars at large anti-centre distances. The photometric data are contained in Table 1 together with 
those for the star S285-6, which has been studied previously by Rolleston et al. (1994).

Table 1. Photometric details of the programme stars

\begin{tabular}{lccccc}
\hline \hline Star & SpT & $V$ & $B-V$ & $U-B$ & $E(B-V)$ \\
\hline S285-6 & B0 V & 12.05 & 0.26 & -0.65 & 0.56 \\
S208-6 & B1.5 V & 12.65 & 0.63 & -0.31 & 0.93 \\
S289-2 & B0 V & 12.66 & 0.21 & -0.61 & 0.53 \\
S289-4 & B1.5 V & 13.58 & 0.21 & -0.61 & 0.51 \\
S283-2 & B0 V & 14.22 & 0.65 & -0.40 & 0.95 \\
RLWT-13 & B1 V & 13.00 & 0.48 & -0.48 & 0.76 \\
RLWT-41 & B1.5 V & 11.39 & 0.37 & -0.46 & 0.67 \\
\hline
\end{tabular}

The observational data have been gathered during six different observing runs and one service night, and a summary of the observations can be found in Table 2. The three stars in the clusters S208 and S289 were observed in December 1991 using the $4.2 \mathrm{~m}$ William Herschel Telescope (WHT) on La Palma in the Canary Islands. We employed the ISIS spectrograph, using the $5300 \AA$ dichroic with gratings $\mathrm{H} 2400 \mathrm{~B}$ and $\mathrm{R} 1200 \mathrm{R}$ on the blue and red arms respectively. Two large format $(1279 \times 1179$ pixels $)$ coated EEV CCDs were used to give a full-width-halfmaximum $(\mathrm{FWHM})$ spectral resolution of $\sim 0.4 \AA$ in the blue and $\sim 0.8 \AA$ in the red.

A subsequent run on the WHT in November 1993 produced data for S283-2. However due to poor weather conditions, the data set was not complete and further observations of this star were thus sought. A service night on the WHT in October 1994 produced additional material and the observations were completed at the same telescope in January 1995. In all three cases the instrumental setup was identical; ISIS spectrograph together with the 5300 $\AA$ dichroic and H2400B and R1200R gratings. The CCD detectors used were TEK1 $(1124 \times 1124$ pixels $)$ and TEK2 $(1124 \times 1124)$ on the blue and red arms respectively with similar spectral resolutions as in the earlier observations. In all cases the spectral regions were primarily optimised to contain Balmer line profiles (which are indicators of surface gravity) and lines of different ionization stages of silicon (the ratio of which constrain the effective temperature of the star). They also contain helium and metal line absorption features typical of early-type stars.

During another observing run on the WHT (February 1993) using the Utrecht Échelle Spectrograph (UES) in conjunction with the 79 lines $\mathrm{mm}^{-1}$ grating and the TEK1 CCD, we observed the stars RLWT-41 and RLWT13. The spectral resolution provided by this instrument was typically better than $0.1 \AA$ FWHM. However as our stars have a projected rotational velocity of greater than $40 \mathrm{~km} \mathrm{~s}^{-1}$, corresponding to a FWHM of $\geq 0.9 \AA$, we were able to bin the spectra to give a similar resolution to that provided by ISIS i.e. $0.4 \AA$. This considerably increased the signal-to-noise of the spectra without degrading the required spectral resolution. Two grating settings were employed, centred on $4273 \AA$ and $4239 \AA$ giving virtually complete coverage between $3860 \AA-4830 \AA$. The typical wavelength range of one échelle order is approximately $40 \AA$, making it extremely difficult to normalise an order containing a $\mathrm{H}$ I line due to the lack of extensive continuum regions. Hence further suitable observations of $\mathrm{H} \delta$ and/or $\mathrm{H} \gamma$ were sought using a system which provided greater wavelength coverage. RLWT-41 was observed in October 1994 with the Jacobus Kaptyen Telescope (JKT) on La Palma using the Richardson-Brearely Spectrograph (RBS). We used the 2400 lines $\mathrm{mm}^{-1}$ grating and the TEK4 CCD to give a FWHM spectral resolution of $\sim 1.0 \AA$ A. RLWT-13 was observed at the Coudé focus of the 107 inch McDonald telescope using a TEK CCD with a spectral resolution of $0.5 \AA$ FWHM.

The observation and reduction techniques were essentially the same for both the single order and the échelle datasets, and as they are similar to those described previously (see for example Rolleston et al. 1994 and references therein), will only be outlined briefly here. The observations were divided into exposures of 1200 or 1500 seconds to reduce the impact of cosmic ray contamination. Short exposures of $\mathrm{Cu}-\mathrm{Ar}$ or $\mathrm{Cu}-\mathrm{Ne}$ arcs were taken between each frame to facilitate wavelength calibration. Bias frames and flat-field exposures were taken at the end of each night. Standard reduction of the 2-dimensional, CCD frames containing the single and multiple order spectra was carried out using the STARLINK package FIGARO (Fuller 1989). The reduced 1-dimensional spectra were analysed using the STARLINK spectral analysis programme DIPSO (Howarth et al. 1995). Normalisation was achieved in each case by fitting a low-order polynomial to continuum regions free from absorption lines.

For all targets, the metal line spectra had a signal-tonoise ratio of greater than $\sim 50$. Equivalent widths for the metal and non-diffuse helium lines were estimated by non-linear least square fitting of single or multiple gaussian profiles to the normalised spectra. The hydrogen and diffuse helium line equivalent widths were not measured but the normalised profiles were extracted directly. Equivalent width measurements for each star can be found in Table 3. The letters $\mathrm{a}, \mathrm{b}$ and $\mathrm{c}$ refer to error estimates in the quoted values with; a: reflecting an error typically less than $10 \%$, b: less than $20 \%$, and c: greater than $20 \%$.

\section{Analysis}

The methods used to determine the atmospheric parameters are effectively identical to those discussed previously in, for example, Dufton et al. (1990) and Rolleston et al. (1993). All theoretical results contained in this study are based on the grid of line-blanketed LTE model atmospheres of Kurucz (1991). 
Table 2. Journal of observations

\begin{tabular}{|c|c|c|c|c|c|}
\hline$\overline{\overline{\text { Object }}}$ & $\overline{\overline{\text { Telescope }}}$ & $\overline{\text { Date }}$ & Instrument & "Resolution & "Spectral Regions \\
\hline \multirow[t]{2}{*}{$\overline{\mathrm{S} 285-6}$} & WHT & Dec. 91 & ISIS & $0.4 \AA$ & $3940-4160 \AA$ \\
\hline & & & & $0.8 \AA$ & $5550-6000 \AA, 6290-6740 \AA$ \\
\hline \multirow[t]{2}{*}{ S208-6 } & WHT & Dec. 91 & ISIS & $0.4 \AA$ & $3940-4160 \AA, 4440-4660 \AA$ \\
\hline & & & & $0.8 \AA$ & $5550-6000 \AA, 6290-6740 \AA$ \\
\hline \multirow[t]{2}{*}{ S289-2 } & WHT & Dec. 91 & ISIS & $0.4 \AA$ & $3940-4160 \AA, 4440-4660 \AA$ \\
\hline & & & & $0.8 \AA$ & $5550-6000 \AA, 6290-6740 \AA$ \\
\hline \multirow[t]{2}{*}{ S289-4 } & WHT & Dec. 91 & ISIS & $0.4 \AA$ & $3940-4160 \AA, 4440-4660 \AA$ \\
\hline & & & & $0.8 \AA$ & $5550-6000 \AA, 6290-6740 \AA$ \\
\hline \multirow[t]{6}{*}{ S283-2 } & WHT & Nov. 93 & ISIS & $0.4 \AA$ & $3972-4177 \AA, 4446-4670 \AA$ \\
\hline & & & & $0.8 \AA$ & $5515-6022 \AA, 6270-6782 \AA$ \\
\hline & & Oct. 94 & ISIS & $0.4 \AA$ & $3972-4177 \AA$ \\
\hline & & & & $0.8 \AA$ & 5515-6022 ̊ \\
\hline & & Jan. 95 & ISIS & $0.4 \AA$ & $4446-4670 \AA$ \\
\hline & & & & $0.8 \AA$ & $6270-6782 \AA$ \\
\hline \multirow[t]{2}{*}{ RLWT-13 } & WHT & Feb. 93 & UES & $0.1 \AA$ & $3855-4945 \AA$ \\
\hline & 2.7m McDonald & Nov. 94 & Coudé & $0.5 \AA$ & 4278-4404 $\AA$ \\
\hline \multirow[t]{2}{*}{ RLWT-14 } & WHT & Feb. 93 & UES & $0.1 \AA$ & $3855-4945 \AA$ \\
\hline & JKT & Oct. 94 & RBS & $1.0 \AA$ & $4000-4435 \AA$ \\
\hline
\end{tabular}

\subsection{Stellar atmospheric parameters}

To ensure that the analysis is as consistent as possible we have used the same techniques to estimate the atmospheric parameters of each star. The programme stars and their comparison, S285-6, all have effective temperatures between $25000 \mathrm{~K}-29000 \mathrm{~K}$, allowing the effective temperature to be deduced from the ionization balance of $\mathrm{Si}$ III/Si IV. The Si III 4567.8, 4574.8, and the Si IV 4116.1 $\AA$ lines were measurable in all the stellar spectra, and primarily only the relative strengths of these lines were considered when estimating the effective temperature. In cases where other lines of silicon were measured in the spectra, it was found that their line strengths were consistent with these estimates, and hence that their inclusion did not significantly alter our results. Surface gravities were deduced by fitting the Balmer lines with theoretical profiles calculated using the line broadening theory of Vidal et al. (1973). The wavelength range for the ISIS spectra included the $\mathrm{H} \epsilon, \mathrm{H} \delta$, and $\mathrm{H} \alpha$ lines. Due to blending with $\mathrm{He}$ I and interstellar calcium, $\mathrm{H} \epsilon$ was ignored; $\mathrm{H} \alpha$ was also not considered because of the predicted larger nonLTE effects for this line (Auer \& Mihalas 1972); indeed in our analysis it consistently gave surface gravity estimates much higher than that for $\mathrm{H} \delta$ and $\mathrm{H} \gamma$. For RLWT41 both $\mathrm{H} \delta$ and $\mathrm{H} \gamma$ were observed and used in the analysis while for RLWT-13, only $\mathrm{H} \gamma$ was available. The effective temperature estimates depend to some extent on adopted gravity, hence the simultaneous determination of both involves an iterative process. However the number of iterations is generally small as the ionization equilibria and the Balmer lines are relatively insensitive to gravity and effective temperature respectively.

The adopted atmospheric parameters are listed in Table 4 for each star with estimated random errors of $\pm 1000 \mathrm{~K}$ and \pm 0.2 dex. For S285-6, Rolleston et al. (1994) derived values of $T_{\text {eff }}=28500 \mathrm{~K}$ and $\log g=4.05$; however the equilibria of carbon and nitrogen were included in their analysis due to the high quality of the data set, and the surface gravity was a weighted mean of that derived from $\mathrm{H} \epsilon, \mathrm{H} \delta$, and $\mathrm{H} \alpha$. We have therefore re-analysed this star using the same techniques and spectral features listed above and it is these values that are listed in Table 4.

\subsection{Microturbulence}

To estimate the microturbulence present in the stellar atmospheres, it is necessary to have a large number of lines of the same species available for measurement (Lennon et al. 1988). Normally the best candidate is O II and only the spectra of RLWT-13, with 30 unblended O II lines, possesses a sufficient number to allow a feasible determination of microturbulent velocity. A value of $8 \mathrm{~km} \mathrm{~s}^{-1}$ was found which is slightly higher than the estimate of $5 \mathrm{~km} \mathrm{~s}^{-1}$, found to be appropriate to main-sequence B- 
Table 3. Equivalent widths ( $\mathrm{m} \AA$ ) for target and comparison stars

\begin{tabular}{|c|c|c|c|c|c|c|c|c|}
\hline \multirow[t]{2}{*}{ Line } & \multirow[t]{2}{*}{ Species } & \multicolumn{6}{|c|}{ Star } & \multirow[b]{2}{*}{ S285-6 } \\
\hline & & S208-6 & S289-2 & S289-4 & RLWT-13 & RLWT-41 & S283-2 & \\
\hline$\overline{3864.43}$ & $\mathrm{O}_{\text {II }}$ & - & - & - & $51 \mathrm{c}$ & - & - & - \\
\hline 3864.67 & O II & & & & & & & \\
\hline 3867.48 & He I & - & - & - & $146 \mathrm{a}$ & $144 \mathrm{a}$ & - & - \\
\hline 3882.19 & O II & - & - & - & $41 \mathrm{c}$ & - & - & - \\
\hline 3911.96 & O II & - & - & - & $76 \mathrm{~b}$ & $32 \mathrm{~b}$ & - & - \\
\hline 3912.11 & $\mathrm{O}$ II & & & & & & & \\
\hline 3918.98 & $\mathrm{C}_{\text {II }}$ & - & - & - & $95 \mathrm{a}$ & $82 \mathrm{~b}$ & - & - \\
\hline 3919.01 & $\mathrm{~N}_{\text {II }}$ & & & & & & & \\
\hline 3919.28 & O II & & & & & & & \\
\hline 3919.28 & S III & - & - & - & $39 \mathrm{c}$ & $76 \mathrm{~b}$ & - & - \\
\hline 3920.68 & $\mathrm{C}_{\text {II }}$ & & & & & & & \\
\hline 3945.05 & O II & $59 \mathrm{~b}$ & $40 \mathrm{c}$ & - & $38 \mathrm{c}$ & $31 \mathrm{c}$ & - & $58 \mathrm{a}$ \\
\hline 3954.37 & O II & $64 \mathrm{~b}$ & $26 \mathrm{c}$ & $72 \mathrm{~b}$ & - & $38 \mathrm{c}$ & - & $87 a$ \\
\hline 3964.73 & He I & $197 \mathrm{c}$ & $97 \mathrm{c}$ & $107 \mathrm{c}$ & $112 \mathrm{c}$ & - & - & $154 \mathrm{a}$ \\
\hline 3982.72 & O II & $57 \mathrm{~b}$ & $36 \mathrm{c}$ & $75 \mathrm{~b}$ & $70 \mathrm{~b}$ & $48 \mathrm{c}$ & $36 \mathrm{c}$ & $67 a$ \\
\hline 3983.77 & S III & $59 \mathrm{~b}$ & - & - & - & - & - & - \\
\hline 3985.97 & S III & $32 \mathrm{~b}$ & - & - & - & - & - & - \\
\hline 3995.00 & $\mathrm{~N}_{\text {II }}$ & $57 \mathrm{a}$ & $24 \mathrm{c}$ & $49 \mathrm{c}$ & $56 \mathrm{c}$ & $54 \mathrm{c}$ & $55 \mathrm{~b}$ & $58 \mathrm{~b}$ \\
\hline 4035.09 & $\mathrm{~N}_{\text {II }}$ & - & - & - & & & $41 \mathrm{c}$ & $30 \mathrm{c}$ \\
\hline 4035.09 & O II & & & & & & & \\
\hline 4041.31 & O II & $37 \mathrm{c}$ & $18 \mathrm{c}$ & - & $51 \mathrm{c}$ & $18 \mathrm{c}$ & - & $34 \mathrm{~b}$ \\
\hline 4041.32 & $\mathrm{~N}_{\text {II }}$ & & & & & & & \\
\hline 4043.54 & $\mathrm{~N}_{\text {II }}$ & - & $<24$ & - & - & - & - & $16 \mathrm{c}$ \\
\hline 4060.58 & O II & - & - & - & - & - & $50 \mathrm{c}$ & $66 \mathrm{a}$ \\
\hline 4060.98 & O II & & & & & & & \\
\hline 4067.94 & $\mathrm{C}_{\text {III }}$ & - & - & - & - & - & $<24$ & $21 \mathrm{c}$ \\
\hline 4068.91 & $\mathrm{C}_{\text {III }}$ & - & - & - & - & - & $<24$ & $21 \mathrm{c}$ \\
\hline 4069.62 & $\mathrm{O}_{\text {II }}$ & $158 \mathrm{a}$ & $160 \mathrm{a}$ & $145 \mathrm{a}$ & $123 a$ & $87 a$ & $126 \mathrm{a}$ & $208 \mathrm{a}$ \\
\hline 4069.89 & $\mathrm{O}_{\text {II }}$ & & & & & & & \\
\hline 4070.30 & $\mathrm{C}_{\text {III }}$ & & & & & & & \\
\hline 4072.16 & O II & $100 \mathrm{a}$ & $84 a$ & $89 a$ & $67 a$ & $57 \mathrm{~b}$ & $59 \mathrm{a}$ & $109 a$ \\
\hline 4074.52 & $\mathrm{C}_{\text {II }}$ & $44 \mathrm{c}$ & $106 \mathrm{~b}$ & $135 \mathrm{a}$ & $97 \mathrm{a}$ & $110 \mathrm{~b}$ & $<35$ & $30 \mathrm{c}$ \\
\hline 4074.85 & $\mathrm{C}_{\text {II }}$ & & & & & & & \\
\hline
\end{tabular}


Table 3. continued

\begin{tabular}{|c|c|c|c|c|c|c|c|c|}
\hline \multirow[t]{2}{*}{$\overline{\overline{\text { Line }}}$} & \multirow[t]{2}{*}{$\overline{\text { Species }}$} & \multicolumn{6}{|c|}{$\overline{\overline{\text { Star }}}$} & \multirow[b]{2}{*}{$\mathrm{S} 285-6$} \\
\hline & & S208-6 & S289-2 & S289-4 & RLWT-13 & RLWT-41 & S283-2 & \\
\hline 4075.85 & $\mathrm{C}_{\text {II }}$ & $133 \mathrm{a}$ & -1 & -1 & -1 & -1 & $97 \mathrm{a}$ & $122 \mathrm{a}$ \\
\hline 4075.86 & $\mathrm{O}_{\text {II }}$ & & & & & & & \\
\hline 4075.94 & $\mathrm{C}_{\text {II }}$ & & & & & & & \\
\hline 4078.84 & $\mathrm{O}_{\text {II }}$ & $49 \mathrm{~b}$ & $25 \mathrm{c}$ & - & $25 \mathrm{c}$ & - & $20 \mathrm{c}$ & $46 \mathrm{~b}$ \\
\hline 4083.91 & O II & $33 \mathrm{c}$ & $40 \mathrm{~b}$ & - & - & - & - & $42 \mathrm{~b}$ \\
\hline 4084.65 & $\mathrm{O}_{\text {II }}$ & $45 \mathrm{c}$ & -2 & $<31$ & $47 \mathrm{c}$ & $30 \mathrm{c}$ & $<25$ & $64 \mathrm{a}$ \\
\hline 4085.11 & $\mathrm{O}_{\text {II }}$ & & & & & & & \\
\hline 4087.16 & O II & $18 \mathrm{c}$ & - & - & $31 \mathrm{c}$ & - & $28 \mathrm{c}$ & $38 b$ \\
\hline 4087.35 & $\mathrm{~N}$ II & & & & & & & \\
\hline 4088.85 & Si IV & $52 \mathrm{~b}$ & $106 \mathrm{a}$ & $97 \mathrm{a}$ & $102 \mathrm{a}$ & $34 \mathrm{c}$ & $156 \mathrm{a}$ & $183 \mathrm{a}$ \\
\hline 4089.29 & $\mathrm{O}_{\text {II }}$ & & & & & & & \\
\hline 4092.93 & O II & - & - & - & - & - & $<27$ & $44 \mathrm{~b}$ \\
\hline 4116.10 & Si IV & $44 \mathrm{~b}$ & $53 \mathrm{~b}$ & $36 \mathrm{c}$ & $31 b$ & $21 \mathrm{c}$ & $84 a$ & $81 \mathrm{a}$ \\
\hline 4119.22 & O II & $77 \mathrm{a}$ & -3 & $97 \mathrm{~b}$ & $71 b$ & $49 \mathrm{c}$ & $70 \mathrm{a}$ & $100 \mathrm{a}$ \\
\hline 4120.28 & $\mathrm{O}_{\text {II }}$ & $311 \mathrm{a}$ & $319 \mathrm{a}$ & $301 \mathrm{a}$ & $275 \mathrm{a}$ & $314 \mathrm{a}$ & $265 \mathrm{a}$ & $338 \mathrm{a}$ \\
\hline 4120.58 & $\mathrm{O}_{\text {II }}$ & & & & & & & \\
\hline 4120.81 & He I & & & & & & & \\
\hline 4120.99 & He I & & & & & & & \\
\hline 4121.48 & O II & & & & & & & \\
\hline 4132.81 & O II & $36 \mathrm{c}$ & $43 b$ & $<25$ & $45 \mathrm{~b}$ & - & $26 \mathrm{c}$ & $62 \mathrm{a}$ \\
\hline 4153.10 & $\mathrm{~S}_{\text {II }}$ & $92 \mathrm{a}$ & $110 \mathrm{a}$ & $59 \mathrm{~b}$ & $64 a$ & $78 \mathrm{a}$ & $61 \mathrm{a}$ & $77 a$ \\
\hline 4153.30 & O II & & & & & & & \\
\hline 4168.97 & $\mathrm{He}_{\mathrm{I}}$ & - & - & - & $93 a$ & $81 \mathrm{a}$ & $57 \mathrm{~b}$ & - \\
\hline 4169.22 & O II & & & & & & & \\
\hline 4185.45 & $\mathrm{O}_{\text {II }}$ & - & - & - & $50 \mathrm{a}$ & - & - & - \\
\hline 4189.79 & $\mathrm{O}_{\text {II }}$ & - & - & - & $65 \mathrm{a}$ & - & - & - \\
\hline 4241.78 & $\mathrm{~N}_{\text {II }}$ & - & - & - & - & $38 b$ & - & - \\
\hline 4253.90 & $\mathrm{O}_{\text {II }}$ & - & - & - & $97 a$ & $62 \mathrm{a}$ & - & - \\
\hline 4254.12 & $\mathrm{O}_{\text {II }}$ & & & & & & & - \\
\hline 4267.02 & $\mathrm{C}_{\text {II }}$ & - & - & - & $102 \mathrm{a}$ & $183 \mathrm{a}$ & - & - \\
\hline 4267.27 & $\mathrm{C}_{\text {II }}$ & & & & & & & \\
\hline 4267.71 & $\mathrm{O}_{\text {II }}$ & & & & & & & \\
\hline 4303.84 & O II & - & - & - & $51 \mathrm{~b}$ & - & - & - \\
\hline 4317.14 & $\mathrm{O}_{\text {II }}$ & - & - & - & $89 a$ & $38 \mathrm{~b}$ & - & - \\
\hline 4317.26 & $\mathrm{C}_{\text {II }}$ & & & & & & & \\
\hline 4319.63 & O II & - & - & - & $76 \mathrm{a}$ & $43 \mathrm{~b}$ & - & \\
\hline 4319.93 & $\mathrm{O}_{\text {II }}$ & & & & & & & \\
\hline
\end{tabular}


Table 3. continued

\begin{tabular}{|c|c|c|c|c|c|c|c|c|}
\hline \multirow[t]{2}{*}{$\overline{\overline{\text { Line }}}$} & \multirow[t]{2}{*}{ Species } & \multicolumn{6}{|c|}{ Star } & \multirow[b]{2}{*}{ S285-6 } \\
\hline & & S208-6 & S289-2 & S289-4 & RLWT-13 & RLWT-41 & S283-2 & \\
\hline 4325.75 & $\mathrm{O}_{\text {II }}$ & - & - & - & $34 \mathrm{~b}$ & - & - & - \\
\hline 4347.22 & $\mathrm{O}_{\text {II }}$ & - & - & - & $37 \mathrm{~b}$ & - & - & - \\
\hline 4347.42 & O II & & & & & & & \\
\hline 4349.43 & $\mathrm{O}_{\text {II }}$ & - & - & - & $95 \mathrm{a}$ & $31 b$ & - & - \\
\hline 4351.26 & $\mathrm{O}_{\text {II }}$ & - & - & - & $61 \mathrm{a}$ & $21 \mathrm{c}$ & - & - \\
\hline 4351.50 & O II & & & & & & & \\
\hline 4366.89 & $\mathrm{O}_{\text {II }}$ & - & - & - & $71 \mathrm{a}$ & $37 \mathrm{~b}$ & - & - \\
\hline 4369.27 & O II & - & - & - & $25 \mathrm{c}$ & - & - & - \\
\hline 4374.27 & $\mathrm{C}_{\text {II }}$ & - & - & - & $24 \mathrm{c}$ & - & - & - \\
\hline 4411.20 & $\mathrm{C}_{\mathrm{II}}$ & - & - & - & - & $26 \mathrm{c}$ & - & - \\
\hline 4411.53 & $\mathrm{C}_{\text {II }}$ & & & & & & & \\
\hline 4414.90 & $\mathrm{O}_{\text {II }}$ & - & - & - & $111 \mathrm{a}$ & $67 \mathrm{~b}$ & - & - \\
\hline 4416.97 & $\mathrm{O}_{\text {II }}$ & - & - & - & $90 \mathrm{a}$ & $62 \mathrm{~b}$ & - & - \\
\hline 4437.03 & He I & - & - & - & $98 \mathrm{a}$ & - & - & - \\
\hline 4447.03 & $\mathrm{~N}_{\text {II }}$ & $31 \mathrm{c}$ & $<26$ & - & $36 \mathrm{c}$ & $33 \mathrm{~b}$ & - & $27 \mathrm{c}$ \\
\hline 4448.21 & $\mathrm{O}_{\text {II }}$ & $39 \mathrm{c}$ & - & - & - & - & - & $39 \mathrm{~b}$ \\
\hline 4479.89 & $\mathrm{Al}_{\text {III }}$ & - & - & - & - & - & $<28$ & $20 \mathrm{c}$ \\
\hline 4479.97 & Al III & & & & & & & \\
\hline 4481.13 & Mg II & $105 a$ & $72 \mathrm{a}$ & $80 a$ & $121 \mathrm{a}$ & - & $74 \mathrm{a}$ & $110 \mathrm{a}$ \\
\hline 4481.33 & $\mathrm{Mg}$ II & & & & & & & \\
\hline 4491.23 & O II & - & - & - & - & - & $30 \mathrm{c}$ & $25 \mathrm{c}$ \\
\hline 4512.54 & Al III & - & - & - & $29 \mathrm{c}$ & $33 \mathrm{c}$ & $39 c$ & $35 \mathrm{c}$ \\
\hline 4528.91 & $\mathrm{Al}_{\text {III }}$ & $35 \mathrm{c}$ & - & $49 \mathrm{c}$ & $44 \mathrm{~b}$ & $45 \mathrm{~b}$ & $53 \mathrm{~b}$ & $32 \mathrm{~b}$ \\
\hline 4529.18 & Al III & & - & & & & & \\
\hline 4552.38 & $\mathrm{~S}_{\text {II }}$ & & & & & & & \\
\hline 4552.65 & Si III & $144 \mathrm{a}$ & $95 \mathrm{a}$ & $108 \mathrm{a}$ & - & - & $108 \mathrm{a}$ & $151 \mathrm{a}$ \\
\hline 4567.82 & Si III & $112 \mathrm{a}$ & $67 a$ & $84 a$ & $100 \mathrm{a}$ & $79 \mathrm{~b}$ & $84 a$ & $140 \mathrm{a}$ \\
\hline 4574.86 & Si III & $81 \mathrm{a}$ & $35 \mathrm{c}$ & $28 \mathrm{c}$ & $79 a$ & $46 \mathrm{~b}$ & $50 \mathrm{~b}$ & $86 \mathrm{a}$ \\
\hline 4590.97 & $\mathrm{O}_{\text {II }}$ & $79 a$ & $85 a$ & $68 \mathrm{a}$ & $89 a$ & $41 \mathrm{~b}$ & $69 a$ & $105 a$ \\
\hline 4595.96 & $\mathrm{O}_{\text {II }}$ & $94 \mathrm{a}$ & $64 a$ & $56 \mathrm{~b}$ & $82 a$ & $48 \mathrm{~b}$ & $51 \mathrm{~b}$ & $108 \mathrm{a}$ \\
\hline 4596.17 & O II & & & & & & & \\
\hline 4601.48 & $\mathrm{~N}_{\text {II }}$ & $62 \mathrm{c}$ & - & $42 \mathrm{c}$ & $50 \mathrm{~b}$ & - & $<20$ & - \\
\hline 4602.11 & $\mathrm{O}_{\text {II }}$ & & - & & & & & \\
\hline
\end{tabular}


Table 3. continued

\begin{tabular}{|c|c|c|c|c|c|c|c|c|}
\hline \multirow[t]{2}{*}{ Line } & \multirow[t]{2}{*}{ Species } & \multicolumn{6}{|c|}{ Star } & \multirow[b]{2}{*}{ S285-6 } \\
\hline & & S208-6 & S289-2 & S289-4 & RLWT-13 & RLWT-41 & S283-2 & \\
\hline 4607.16 & $\mathrm{~N}_{\text {II }}$ & - & - & - & $27 \mathrm{c}$ & - & $<20$ & - \\
\hline 4609.44 & O II & $43 \mathrm{~b}$ & $64 \mathrm{~b}$ & $62 \mathrm{a}$ & - & - & $<31$ & $52 \mathrm{c}$ \\
\hline 4610.87 & O II & - & -4 & -4 & - & - & - & $32 \mathrm{c}$ \\
\hline 4613.11 & $\mathrm{O}_{\text {II }}$ & $33 \mathrm{c}$ & - & - & - & - & - & $<16$ \\
\hline 4613.46 & S III & & & & & & & \\
\hline 4613.67 & O II & & & & & & & \\
\hline 4630.54 & $\mathrm{~N}_{\text {II }}$ & $55 \mathrm{~b}$ & -5 & $-{ }^{5}$ & $39 \mathrm{c}$ & $43 \mathrm{~b}$ & - & $<33$ \\
\hline 4631.38 & Si IV & - & $24 \mathrm{~b}$ & $36 \mathrm{c}$ & - & - & - & $<19$ \\
\hline 4638.86 & O II & $98 \mathrm{a}$ & $57 \mathrm{~b}$ & $59 \mathrm{~b}$ & $74 a$ & $42 \mathrm{c}$ & $61 \mathrm{~b}$ & $105 a$ \\
\hline $\begin{array}{l}4641.82 \\
4641.90\end{array}$ & $\begin{array}{l}\mathrm{O}_{\text {II }} \\
\mathrm{N}_{\text {III }}\end{array}$ & $118 \mathrm{a}$ & $109 b$ & $155 \mathrm{a}$ & $96 a$ & $57 \mathrm{c}$ & $102 a$ & $147 a$ \\
\hline 4643.09 & $\mathrm{~N}_{\text {II }}$ & $35 \mathrm{~b}$ & -6 & -6 & - & - & $40 \mathrm{~b}$ & $29 \mathrm{c}$ \\
\hline 4647.42 & $\mathrm{C}_{\text {III }}$ & $74 a$ & -7 & $72 \mathrm{a}$ & - & - & $91 \mathrm{a}$ & $118 \mathrm{a}$ \\
\hline 4649.14 & $\mathrm{O}_{\text {II }}$ & $134 \mathrm{a}$ & -7 & -7 & - & - & $91 \mathrm{a}$ & $163 a$ \\
\hline 4650.25 & $\mathrm{C}$ III & $144 \mathrm{a}$ & $353 \mathrm{a}$ & $332 \mathrm{a}$ & - & - & $136 \mathrm{a}$ & $102 \mathrm{~b}$ \\
\hline 4650.84 & $\mathrm{O}_{\text {II }}$ & & & & & & -8 & $108 \mathrm{~b}$ \\
\hline 4651.47 & $\mathrm{C}_{\text {III }}$ & & & & & & $87 a$ & $50 \mathrm{c}$ \\
\hline 4661.63 & O II & - & - & - & $76 \mathrm{~b}$ & $52 \mathrm{~b}$ & - & - \\
\hline 4673.74 & O II & - & - & - & $35 \mathrm{c}$ & - & - & - \\
\hline 4676.24 & O II & - & - & - & $68 \mathrm{~b}$ & $38 \mathrm{c}$ & - & - \\
\hline 4696.35 & O II & - & - & - & $18 \mathrm{c}$ & - & - & - \\
\hline 4699.00 & $\mathrm{O}_{\text {II }}$ & - & - & - & $97 \mathrm{~b}$ & $47 \mathrm{c}$ & - & - \\
\hline 4699.22 & O II & & & & & & & \\
\hline 4703.16 & O II & - & - & - & $21 \mathrm{c}$ & - & - & - \\
\hline 4705.35 & $\mathrm{O}_{\text {II }}$ & - & - & - & $84 a$ & $32 \mathrm{c}$ & - & - \\
\hline 4710.01 & $\mathrm{O}_{\text {II }}$ & - & - & - & $33 \mathrm{c}$ & $15 \mathrm{c}$ & - & - \\
\hline 4713.14 & He I & - & - & - & $232 \mathrm{a}$ & $269 \mathrm{a}$ & - & - \\
\hline 4713.47 & He I & & & & & & & \\
\hline
\end{tabular}


Table 3. continued

\begin{tabular}{|c|c|c|c|c|c|c|c|c|}
\hline \multirow[t]{2}{*}{ Line } & \multirow[t]{2}{*}{ Species } & \multicolumn{6}{|c|}{ Star } & \multirow[b]{2}{*}{ S285-6 } \\
\hline & & S208-6 & S289-2 & S289-4 & RLWT-13 & RLWT-41 & S283-2 & \\
\hline 4716.65 & Si III & - & - & - & $36 \mathrm{c}$ & $23 \mathrm{c}$ & - & - \\
\hline 4813.30 & Si III & - & - & - & $43 \mathrm{c}$ & - & - & - \\
\hline 4819.72 & Si III & - & - & - & $38 \mathrm{c}$ & - & - & - \\
\hline 4828.96 & Si III & - & - & - & $19 \mathrm{c}$ & $19 \mathrm{c}$ & - & - \\
\hline 4906.83 & $\mathrm{O}_{\text {II }}$ & - & - & - & $51 \mathrm{c}$ & - & - & - \\
\hline 5666.64 & $\mathrm{~N}$ II & $35 \mathrm{c}$ & - & $31 b$ & - & - & - & - \\
\hline 5679.56 & $\mathrm{~N}_{\text {II }}$ & $52 \mathrm{~b}$ & $<19$ & $58 \mathrm{~b}$ & - & - & $50 \mathrm{a}$ & $45 \mathrm{c}$ \\
\hline 5696.00 & $\mathrm{C}_{\text {III }}$ & $65 a$ & - & $62 a$ & - & - & $118 \mathrm{a}$ & $97 \mathrm{a}$ \\
\hline 5696.47 & $\mathrm{Al}$ III & & & & & & & \\
\hline 5710.76 & $\mathrm{~N}_{\text {II }}$ & - & - & - & - & - & $35 a$ & - \\
\hline 5722.65 & AlıII & $36 \mathrm{c}$ & - & $43 \mathrm{~b}$ & - & - & $13 \mathrm{~b}$ & $43 \mathrm{c}$ \\
\hline 5739.76 & Si III & $79 a$ & - & $56 \mathrm{a}$ & - & - & $56 \mathrm{a}$ & $99 \mathrm{a}$ \\
\hline 5940.25 & $\mathrm{~N}_{\text {II }}$ & $99 \mathrm{a}$ & - & $91 \mathrm{a}$ & - & - & $98 \mathrm{a}$ & $97 \mathrm{c}$ \\
\hline 5940.69 & $\mathrm{~S}_{\text {II }}$ & & & & & & & \\
\hline 5941.67 & $\mathrm{~N}_{\text {II }}$ & & & & & & & \\
\hline 6482.07 & $\mathrm{~N}_{\text {II }}$ & - & - & $52 \mathrm{~b}$ & - & - & $<33$ & $42 \mathrm{c}$ \\
\hline 6578.03 & $\mathrm{C}_{\mathrm{II}}$ & $73 \mathrm{c}$ & $27 \mathrm{c}$ & $74 \mathrm{c}$ & - & - & $73 a$ & $71 \mathrm{c}$ \\
\hline 6582.85 & $\mathrm{C}_{\mathrm{II}}$ & $56 \mathrm{c}$ & - & $71 \mathrm{c}$ & - & - & - & $48 \mathrm{c}$ \\
\hline 6721.35 & O II & $49 \mathrm{c}$ & $26 \mathrm{c}$ & $27 \mathrm{c}$ & - & - & $<12$ & $68 \mathrm{c}$ \\
\hline
\end{tabular}

1. - Included in equivalent width for $\mathrm{C}$ I lines at approximately $4074 \AA$.

2. - Included in equivalent width for $\mathrm{O}_{\text {II }}$ line at $4083.91 \AA$.

3. - Included in equivalent width for lines approximately at $4120 \AA$.

4. - Included in equivalent width for $\mathrm{O}$ is line at $4609.44 \AA$.

5. - Included in equivalent width for Si Iv line at $4631.83 \AA$

6. - Included in equivalent width for lines approximately at $4640 \AA$.

7. - Included in equivalent width for lines approximately at $4650 \AA$.

8. - Included in equivalent width for $\mathrm{C}_{\text {III }}$ line at $4650.25 \AA$.

star atmospheres from the LTE analyses of Hardorp \& Scholz (1970), Peters (1976) and Dufton et al. (1990). Assuming this value of $5 \mathrm{~km} \mathrm{~s}^{-1}$ would increase our derived abundances by typically 0.1 dex and always by less than 0.2 dex.

It was not possible to measure enough $\mathrm{O}$ II lines in the five spectra of the other programme stars to make a mean- ingful estimation of the microturbulence and hence a value of $5 \mathrm{~km} \mathrm{~s}^{-1}$ was chosen. Deviations of up to $5 \mathrm{~km} \mathrm{~s}^{-1}$ in the adopted value would alter our mean abundances by typically between $0.1-0.2 \mathrm{dex}$, and our principal conclusions are not compromised by this assumption. Rolleston et al. (1994) derived a value of $6 \mathrm{~km} \mathrm{~s}^{-1}$ for S285-6, and details can be found therein. 
Table 4. Physical properties of the programme stars

\begin{tabular}{lccccc}
\hline \hline Star & $T_{\text {eff }}$ & $\log g$ & $v_{\mathrm{t}}$ & $\operatorname{Mass}\left(M_{\odot}\right)$ & $V \sin i\left(\mathrm{~km} \mathrm{~s}^{-1}\right)$ \\
\hline S208-6 & 26000 & 4.0 & 5 & 12 & 31 \\
S289-2 & 28500 & 4.0 & 5 & 13 & 64 \\
S289-4 & 27000 & 4.1 & 5 & 12 & 50 \\
RLWT-41 & 26000 & 4.25 & 5 & 10 & 51 \\
RLWT-13 & 25000 & 4.0 & 8 & 10 & 41 \\
S283-2 & 29000 & 3.9 & 5 & 15 & 25 \\
S285-6 & 27500 & 4.0 & 6 & 13 & 20 \\
\hline
\end{tabular}

\subsection{Chemical Composition}

For each star we generated LTE model atmospheres with the atmospheric parameters as quoted in Table 4. Helium and metal abundances were estimated from comparing observed line strengths and profiles with those derived from our theoretical grids. The atomic data were as discussed in Jeffery (1991). Only those metal and non-diffuse helium lines, that we believed were essentially unblended, were used in this study. The strengths of the diffuse helium lines were not measured but the profiles were fitted directly, allowing a further determination of the helium abundance.

Photospheric abundance estimates are listed in Table 5 , where abundances are given in a logarithmic form on the scale hydrogen $=12.0$ dex. The values are simple means of the results from individual equivalent widths, ignoring those lines which have high observational uncertainties. The errors (where quoted) are the standard deviations from the mean value in each case. In the cases were a large number of individual lines are available, and if the errors are randomly distributed, then the expected error in the mean abundance should be less than those quoted.

\section{Discussion of results}

\subsection{Distance estimates and cluster membership}

It is important to determine reliable distances to the programme stars and compare these estimates with those calculated previously using different methods e.g Moffat et al. (1979). We must also confirm that the stars designated by a Sharpless number are indeed associated with the appropriate $\mathrm{H}$ II region.

Our method of calculating distances from the atmospheric parameters and photometric measurements (listed in Sect. 2) was as follows. Masses were derived by placing the stars in the effective temperature - surface gravity diagram using the evolutionary tracks of Maeder \& Meynet (1988). Using more recently published grids of stellar evolutionary models, such as Schaller et al. (1992), does not significantly alter the inferred masses. This allowed a calculation of the luminosity which is reflective of the stars current position in the HR-diagram, rather than assuming that the object is on the zero-age main sequence (ZAMS). The absolute visual magnitude was then determined employing the bolometric corrections of Kurucz (1979). We calculated the distance using the $E(B-V)$ listed in Table 1 and assuming a standard galactic extinction law (Seaton 1979). The individual clusters and field stars are discussed below.

S208 :- Our target although called S208-6 following the nomenclature of Moffat et al. (1979), is probably associated with a faint, small region of emission nebulosity Wat 1 . This association is relatively close on the sky to S208 ( $5^{\prime}$ away) but appears to be at a smaller distance. Moffat et al. conclude that it is probably fortuitous that both exist along similar lines of sight since the distances determined by zero-age main sequence fitting are $7.6 \mathrm{kpc}$ (S208) and $4.4 \mathrm{kpc}$ (Wat 1). Our method yields an estimate of $3.4 \mathrm{kpc}$ which, although smaller than that of Moffat et al. may not be inconsistent, given our error estimates of $\pm 1 \mathrm{kpc}$ (mainly arising from the uncertainty of \pm 0.2 dex in surface gravity).

S289 :- This cluster is associated with a weak extended $\mathrm{H}$ II region and with $b=-4.6^{\circ}$ is at a significant distance below the galactic plane. Its low mean reddening is compatible with its expected line of sight extinction at this latitude. Both methods of ZAMS fitting of cluster members and spectroscopic parallaxes (Moffat et al. 1979) yield a distance of $7.9 \mathrm{kpc}$. This is in good agreement with our mean estimate of $7.8 \mathrm{kpc}$. At this distance the cluster would be $630 \mathrm{pc}$ below the plane. This is not unusual given that the thickness of the disk at these distances, as mapped by neutral hydrogen, is observed to be considerably broader than regions within the solar circle (Burton 1976).

S283 :- Again good agreement is found between our calculation and the estimate of Moffat et al. The ZAMS fitting produces a value of $9.1 \mathrm{kpc}$, which compares well with our estimate of $9.7 \mathrm{kpc}$, making this the most distant cluster in this study. The star S283-2 shows a greater reddening than others as it is embedded in a dense core of the $\mathrm{H}$ il region.

RLWT-13 :- The two RLWT stars are not physically related and are probably not members of any particular OB associations. A distance of $7.1 \mathrm{kpc}$ to RLWT-13, 

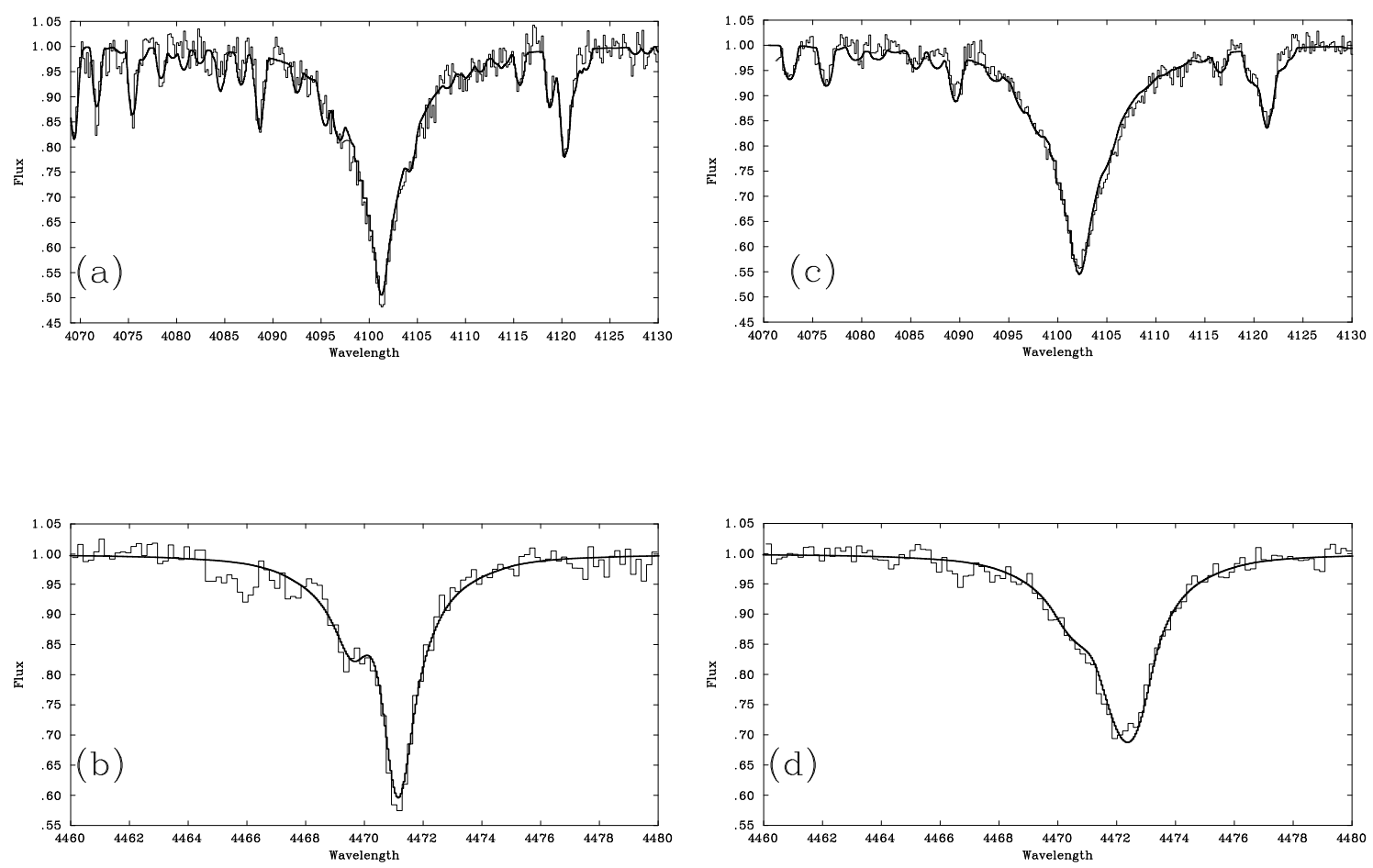

Fig. 1. Examples of observed spectral regions (histograms) obtained for two of the programme stars including a) H $\delta$ from S208-6; b) He I $4471 \AA$ from S208-6; c) H $\delta$ from S289-2; d) He I $4471 \AA$ from S289-2. Also shown are the theoretical spectra (bold smooth curves) which have been calculated using the atmospheric parameters in Table 3 and the abundances in Table 4 . Good agreement is found between theory and observation

Table 5. Logarithmic helium and metal abundances

\begin{tabular}{rllllllll}
\hline \hline Star & He I & C II & N II & O II & Mg II & Al III & Si III & Si IV \\
\hline S208-6 & $10.98 \pm 0.17$ & $7.46 \pm 0.05$ & $7.40 \pm 0.12$ & $8.59 \pm 0.15$ & 7.23 & $5.98 \pm 0.12$ & $7.23 \pm 0.05$ & 7.27 \\
S289-2 & $10.99 \pm 0.15$ & 7.08 & 7.02 & $8.30 \pm 0.11$ & 7.14 & - & $6.74 \pm 0.01$ & 6.74 \\
S289-4 & $10.99 \pm 0.16$ & $7.68 \pm 0.14$ & $7.56 \pm 0.08$ & $8.36 \pm 0.13$ & 7.08 & - & $6.72 \pm 0.2$ & 6.80 \\
RLWT-13 & $10.88 \pm 0.12$ & - & $7.31 \pm 0.16$ & $8.53 \pm 0.19$ & 7.20 & $5.84 \pm 0.04$ & $7.01 \pm 0.14$ & 6.98 \\
RLWT-41 & $10.95 \pm 0.09$ & - & $7.39 \pm 0.12$ & $8.00 \pm 0.17$ & - & 6.10 & $6.74 \pm 0.14$ & 6.78 \\
S283-2 & $11.03 \pm 0.05$ & 7.83 & $7.75 \pm 0.21$ & $8.28 \pm 0.17$ & 7.11 & $6.31 \pm 0.22$ & $7.10 \pm 0.03$ & 7.10 \\
S285-6 & $10.96 \pm 0.11$ & $7.69 \pm 0.18$ & $7.45 \pm 0.19$ & $8.58 \pm 0.18$ & 7.38 & $6.04 \pm 0.27$ & $7.35 \pm 0.06$ & $7.39 \pm 0.03$ \\
\hline
\end{tabular}

calculated by Philip et al. (1990) by using an absolutemagnitude - spectral type relation is significantly different to our estimate of $4.6 \mathrm{kpc}$. The absolute magnitude used by Philip et al. of $M_{v}=-3.58$ may be an over-estimate as our model atmospheric analysis indicates that this star is close to the ZAMS, and hence a lower intrinsic luminosity is probably more appropriate. The main source of error in our calculation is again uncertainties in the logarith- mic surface gravity, with a decrease of 0.2 dex typically causing an increase of $30 \%$ in the distance.

RLWT-41 :- Similar to RLWT-13, we find that our distance estimate $(1.9 \mathrm{kpc})$ is significantly less than that quoted by Philip et al. of $3.2 \mathrm{kpc}$. The estimated logarithmic surface gravity of 4.25 dex places the star on or very close to the ZAMS and produces a lower value of calculated absolute visual magnitude than that assumed in the photometric estimation $\left(M_{v}=-3.58\right)$. Again we believe 
that our method yields a more reliable distance due to the fact that we are calculating a luminosity appropriate to the stars' current position in the Hertzprung - Russell diagram.

A full discussion of the galactic positions of our stars which considers the spiral arm structure at large galactocentric distances and sources of systematic errors in the distance determinations is contained in Paper II.

\subsection{Metal abundances}

The primary aim of these papers is to meaningfully compare metal abundances of B-stars, with similar atmospheric parameters, which lie at different spatial positions and galactocentric distances within the Galaxy in the anticentre direction. We emphasize that our absolute abundances listed in Table 5 may not be as reliable as those deduced by using non-LTE techniques (see for example Gies \& Lambert 1992; Kilian-Montenbruck et al. 1996). However by only considering targets with very similar atmospheric parameters and using consistent data reduction and analysis techniques, we expect our differential results to be highly reliable.

The star S285-6 provides an ideal comparison to suitably contrast the abundance variations displayed by our programme stars, as its observational dataset is of an exceptionally high quality; its spectra have a continuum signal-to-noise ratio of $\sim 100$ and being a sharp-lined star it was possible to measure equivalent widths down to $\sim 20 \mathrm{~m} \AA$. Rolleston et al. (1994) reported the absolute abundances of the open cluster associated with the H II region $\mathrm{S} 285$ based on the photospheric abundances of two B0 V cluster members (including S285-6) and concluded that the derived abundances closely reflected those of Bstars within the solar neighbourhood (cf. Fitzsimmons et al. 1990). Additionally the element abundances from the two stars were in good internal agreement with the possible exception of nitrogen. We have re-analysed the observational data for S285-6 using our atmospheric parameters (as discussed in Sect. 3.1) and it is these results that are quoted in Table 5. The results from our abundance analysis do not deviate significantly from those quoted by Rolleston et al. apart from perhaps the nitrogen abundance which, from $6 \mathrm{~N}$ II lines, we estimate to be 0.1 dex lower. Rolleston et al. suggested that $\mathrm{S} 285-6$ showed a significantly different nitrogen composition to that of S285-1 and we note these variations when comparing this with the nitrogen abundances of our target stars.

The helium abundance in all the stars appears normal, and there is no evidence for any significant deviations from the abundance found in B-stars in the solar neighbourhood. This estimation is primarily based on the He I 4009, $4026 \AA$ diffuse lines, and in certain cases the $4471 \AA$ line. All of these are well observed and the theoretical profiles match the observations closely, hence we believe our estimates are accurate to \pm 0.1 dex. However our results indicate that significant variations in metal abundances exist between the programme stars. As all our stars have similar atmospheric parameters $\left(25000<T_{\text {eff }}<29000\right.$ $\mathrm{K} ; \log g \sim 4.0 \mathrm{dex})$ we believe that these differences are almost certainly real.

The abundances in S208-6 are generally similar to those found in S285-6. The distance to this cluster of $3.4 \mathrm{kpc}$ confirms the previously found results of Rolleston et al. (1994 \& 1993) that clusters showing near solar chemical compositions exist out to a distance of approximately 12 kpc. Kaufer et al. (1994), in a study of 16 B-type main-sequence stars in young stellar clusters observed and analysed this star, again using line-blanketed model atmosphere techniques. Their derived atmospheric parameters $\left(\mathrm{T}_{\text {eff }}=26500 \mathrm{~K}\right.$, and $\left.\log g=4.10\right)$ are similar to ours and there is good agreement between the metal abundances of the two datasets. A discrepancy however exists in the nitrogen abundance, with values of 7.75 and 7.40 dex from Kaufer et al. and this study respectively. The datasets do not significantly overlap, with only one line (at $4630 \AA$ ) appearing in both studies and the two equivalent width estimates agree to within $10 \%$. The reasons for the differences in the nitrogen abundances are thus unclear since the disparity of 0.35 dex is too large to be due to random statistical errors associated with line strength measurement. The assumption of LTE is the most probable explanation, and while this may cast a doubt over our absolute abundances, any systematic sources of error in our methods will be removed in a differential abundance analysis (see Paper II). Inconsistencies of 0.3 dex also exist for the magnesium abundance. However this estimate is only based on one absorption feature and measurement uncertainties may account for this difference as the equivalent widths from each study differ by $30 \%$.

A different star in the cluster S289 (number 13 in the listing of Moffat et al. 1979) was analysed by KilianMontenbruck et al. (1996) using LTE line blanketed model atmospheres and NLTE line formation techniques (for C, $\mathrm{N}, \mathrm{O}, \mathrm{Mg}, \mathrm{Al}, \& \mathrm{Si}$ ). Significant differences exist between the $\mathrm{N}$ II and C II abundances derived for this star and those listed here for S289-2 and S289-4. Our dataset for these lines is restricted due to the signal-to-noise of the spectra and the moderate rotational velocity of the two stars; hence it is difficult to definitively attribute these differences to real inhomogeneities in the cluster. Additionally the C II lines suffer significantly from NLTE effects (Eber \& Butler 1988) which may explain the large discrepancies found for this species. The abundances found for oxygen, magnesium and aluminium show good agreement in the two datasets with differences similar to, or smaller than, the quoted errors in the two studies. The silicon abundance estimates also show a significant difference, with our results predicting a value 0.6 dex lower than that from Kilian-Montenbruck et al. This may reflect systematic errors due to the different LTE/NLTE 
treatments of these lines although non-LTE effects for both Si III and Si IV ions are believed relatively small (Becker \& Butler 1990). Additionally our silicon abundances are consistent with the moderate depletions found for other metals. A similar discrepancy is found for the stars S285-6 and S285-1 analysed by Kilian-Montenbruck et al. and Rolleston et al. (1994), with the LTE treatment producing an abundance lower by $0.4 \mathrm{dex}$ in this case. The fact that the absolute abundances are so dependent on the method of analysis highlights the need for differential analyses of stars with similar atmospheric parameters.

The abundances derived for S283-2, the most distant star in the sample, appear to show deficiencies in oxygen, magnesium and silicon of the order $0.2-0.3 \mathrm{dex}$, whereas nitrogen and aluminium appear only slightly overabundant. Finally, RLWT-13 appears to show mild deficiencies in each element abundance apart from $\mathrm{O}$ II whereas RLWT-41 exhibits what seems to be significant deficiencies in oxygen and silicon.

The comparisons of the absolute abundances derived for the programme stars and clusters both from this analysis and those of other authors emphasize the need for a detailed line by line differential abundance analysis to remove or at least mitigate the effects of systematic errors in our methods. Additionally, the errors in our distance calculations must be quantified and galactic positions of our stars related to the spiral arm structure at these radii. These problems will be addressed and discussed in Paper II.

Acknowledgements. Observational data were obtained at the William Herschel Telescope and the Jacobus Kaptyen Telescope on the island of La Palma operated by the Royal Greenwich Observatory. We are grateful to the staff for their assistance at the Spanish Observatorio del Roque de los Muchachos of the Instituto de Astrophysica de Las Canarias. Data reduction was performed on the PPARC funded Northern Ireland STARLINK node, and some of the model atmosphere programs were made available through the PPARC supported Collaborative Computational Project No. 7. SJS holds a post-graduate studentship from the Department of Education for Northern Ireland, and would like to thank Nigel Hambly and Eamon
Little for advice and Paul Brown and Cathy Ramsbottom for assistance in obtaining some of the observations.

\section{References}

Auer L.H., Mihalas D., 1972, ApJS 24, 193

Becker S.R., Butler K., 1990, A\&A 235, 326

Burton W.B., 1976, Ann. Rev. Astron. Astrophys. 14, 75

Dufton P.L., Brown P.J.F., Fitzsimmons A., Lennon D.J., 1990, A\&A 232, 431

Eber F., Butler K., 1988, A\&A 202, 153

Fitzsimmons A., Brown P.J.F., Dufton P.L., Lennon D.J., 1990, A\&A 232, 437

Fuller N.M.J., 1989 Starlink User Notice No. 86

Gies D.R., Lambert D.L., 1992, ApJ 387, 673

Hardorp J., Scholz M., 1970, ApJ 154, 1111

Howarth I.D., Murray J., Mills D., Berry D.S., 1995, Starlink User Notice, No. 50.16

Jeffery C.S., 1991, in: Newslett. Anal. Astron. Spectra 16, p. 17

Kaufer A., Szeifert Th., Krenzin R., Baschek B., Wolf B., 1994, A\&A 289, 740

Kilian-Montenbruck J., Gehren T., Nissen N.E., 1996, A\&A (in press)

Kurucz R.L., 1979, ApJS 40, 1

Kurucz R.L., 1991, in Precision Photometry: Astrophysics of the Galaxy. In: Davis-Philip A.G., Upgren A.R., Janes P.L. (eds.). Schenectady: Davis

Lennon D.J., Brown P.J.F., Dufton P.L., 1988, A\&A 195, 208

Maeder A., Meynet G., 1988, A\&AS 76, 411

Moffat A.F.J., Fitzgerald M.P., Jackson P.D., 1979, A\&AS 38, 197

Peters G.J., 1976, ApJS 30, 551

Philip A.G.D., Chromey F.R., Dubois P., 1990, PASP 102, 654

Rolleston W.R.J, Brown P.J.F., Dufton P.L., Fitzsimmons A., 1993, A\&A 270, 107

Rolleston W.R.J, Dufton P.L., Fitzsimmons A., 1994, A\&A 284,72

Schaller G., Schaerer D., Meynet G., Maeder A., 1992, A\&AS 96,269

Seaton M.J., 1979, MNRAS 187, 73

Sharpless S., 1959, ApJS 4, 2573

Smartt S.J., Dufton P.L., Rolleston W.R.J., 1996, A\&A (in press)

Vidal C.R., Copper J., Smith E.W., 1973, APJS 25, 37 